\title{
Spatial Sound Rendering - A Survey
}

\author{
E. Lakka ${ }^{1}$, A. G. Malamos ${ }^{2}$, K. G. Pavlakis ${ }^{2}$, J. A. Ware ${ }^{1 *}$ \\ ${ }^{1}$ Faculty of Computing, Engineering and Science, University of South Wales, Treforest (United Kingdom) \\ ${ }^{2}$ Media, Networks and Communications Lab Dept. of Informatics Engineering Technological \\ Educational Institute of Crete Heraklion, GR 71004 (Greece)
}

Received 23 January 2018 | Accepted 3 May 2018 | Published 1 June 2018

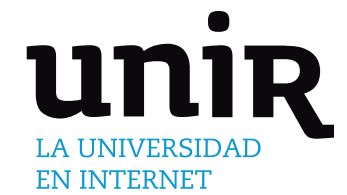

KEYWORDS

ABSTRACT

Simulating propagation of sound and audio rendering can improve the sense of realism and the immersion both in complex acoustic environments and dynamic virtual scenes. In studies of sound auralization, the focus has always been on room acoustics modeling, but most of the same methods are also applicable in the construction of virtual environments such as those developed to facilitate computer gaming, cognitive research, and simulated training scenarios. This paper is a review of state-of-the-art techniques that are based on acoustic principles that apply not only to real rooms but also in 3D virtual environments. The paper also highlights the need to expand the field of immersive sound in a web based browsing environment, because, despite the interest and many benefits, few developments seem to have taken place within this context. Moreover, the paper includes a list of the most effective algorithms used for modelling spatial sound propagation and reports their advantages and disadvantages. Finally, the paper emphasizes in the evaluation of these proposed works.

\author{
Real-time Spatial \\ Audio, Acoustics, 3D \\ Sound, Auralization, \\ Simulation of Sound \\ Propagation, Real-time \\ Systems, Virtual Reality, \\ Immersive Environments. \\ DOI: $10.9781 /$ ijimai.2018.06.001
}

\section{INTRODUCTION}

$\mathrm{T}$ HE last few years a growing interest in virtual environment technologies have been witnessed and their inclusion have been seen in a wide variety of different applications, including distance learning (e.g. serious gaming), the entertainment industry (e.g. online games, live events), architectural design, the production of art, various training scenarios, along with scientific and engineering research [1] [2]. As a result, work documented in the relevant recent literature has focused on improving the realism and the sense of the immersivity within a Three Dimensional (3D) Virtual Environment (VE), taking into account not only the realistic visual rendering but also the spatial sound propagation. The fact that sound rendering constitutes an integral part of the production of realistic VE, is not a coincidence, because it can offer additional details and visceral sense to a 3D immersive world.

Specifically, spatial auditory allows the user of VE to recognise the location of a sound source(s) [3], deduce information of the environment around the sound source(s) and, in general, to conceive the immersive environment in the same way as the listener recognizes the sound in the real world. Additionally, from the physical/algorithmical point of view, sound propagation techniques are used to simulate the sound waves as they travel from each source to the listener by taking into account the interactions with various objects in the scene [4]. In other words, spatial sound rendering in a VE goes far beyond traditional stereo and surround sound techniques, through the estimation of physical attributes, which are involved in sound propagation. Thus,

\section{* Corresponding author.}

E-mail addresses: efilakka@gmail.com (E.Lakka), amalamos@ ie.teicrete.gr (A. G. Malamos), pavlakis@staff.teicrete.gr (K. G. Pavlakis), andrew.ware@southwales.ac.uk (J. A. Ware). characteristics such as surface reflection, diffusion, reverberation, and wave phenomena (interference, diffraction) can be included for the formation of spatial impressions of a virtual 3D scene (more details in Section 1.1).

To summarise, for several years great effort has been devoted to achieve high quality visual rendering for the development of interactive virtual worlds [5].Moreover, considerable attention has been paid to engage multiple senses in $3 \mathrm{D}$ interactive applications, for the reason that it constitutes a vital factor in order to improve the immersion and realism for the user experience [6]. In other words, the 3D VE can be described as a mosaic of technologies that includes visual and auditory rendering in order to simulate the real physical world. Accordingly, the question which arises is whether researchers take into account the attribute of spatial sound propagation for the design of $3 \mathrm{D} \mathrm{VE}$ and what method is used to accomplish the desired result. Additionally, another issue is whether there are similar efforts in web 3D applications, in order to accomplish more sophisticated web virtual environments, with the contribution of the immersive audio.

The objective of this paper is to review empirical research studies and thus facilitate an understanding of the methods which are used in the (web) 3D virtual applications and in room acoustics, in order to render the spatial sound.

The remainder of the paper is organized into the follow sections: Section I.A describes the sound propagation phenomena; Section II analyses the most effective algorithms for the spatial sound propagation and they have been divided into categories; our conclusions are drawn in the final III section.

\section{A. Sound Propagation Phenomena}

This section gives a brief overview of the physical models of sound propagation and other acoustic effects. For the purpose of simulating sound in virtual environments the comprehension of these issues is fundamental. Hence, an explanation of physical phenomena is 
described, in order that the methods and algorithms for realistic sound rendering can be understood.

\section{1) Reflection}

During the propagation of a sound wave in an enclosed space, the wave hits objects or room boundaries and its free propagation is disturbed. Moreover, during this process at least a portion of the incident wave will be thrown back, a phenomenon known as reflection. If the wavelength of the sound wave is small enough in respect to the dimensions of the reflecting object and large compared with possible irregularities of the reflecting surface, a specular reflection occurs. This phenomenon is illustrated in the Fig. 1a, in which the angle of reflection is equal to the angle of incidence. In contrast, if the sound wavelength is comparable with the corrugation dimensions of an irregular reflection surface, the incident sound wave will be scattered in all directions. In this case, the phenomenon is called diffuse reflection (Fig. 1b).

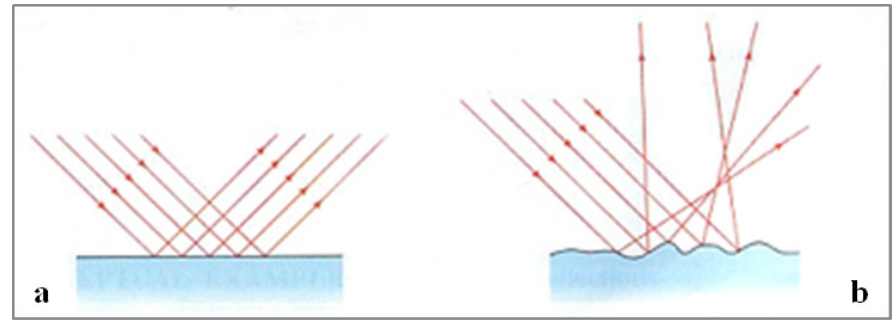

Fig. 1. Reflection - Sound Physical Phenomenon. (a) Specular reflection phenomenon; (b) Diffuse reflection phenomenon.

\section{2) Diffraction}

Another easily experienced characteristic of a sound wave is the diffraction which occurs when, for example, listening to without seeing another person from behind a door. Diffraction is the spread of waves around corners (Fig. 2b), behind obstacles or around the edges of an opening (Fig. 2a). The amount of diffraction increases with wavelength, meaning that sound waves with lower frequencies, and thus with greater wavelengths than obstacles or openings dimensions, will be spread over larger regions behind the openings or around the obstacles [7].

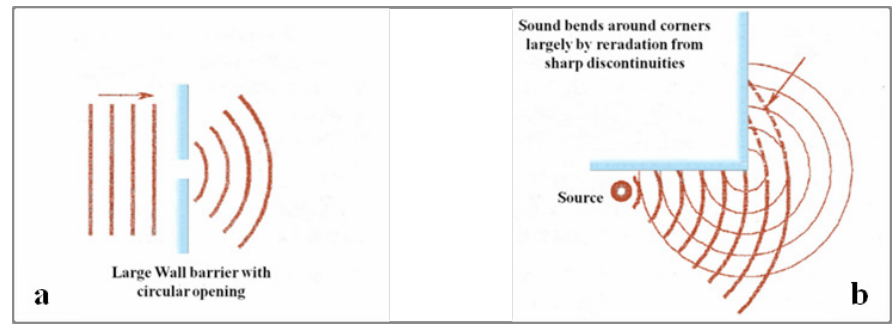

Fig. 2. Diffraction - Sound Physical Phenomenon. (a) Behind obstacles or around the edges of an opening; (b) Around corners.

\section{3) Refraction}

Refraction is the change in the propagation direction of waves when they cross obliquely the boundary between two mediums where their speed is different. This phenomenon should be considered for a realistic sound simulation. The path of a refracted wave can be found using Fermat's principle, which states that sound waves take the path with the least travel time (Fig. 3). For transmission of a plane sound wave from air into another medium, the refraction index in (1) is used, for calculating the geometric conditions [8].

$$
\mathrm{n}=\mathrm{c}^{\prime} / \mathrm{c}=\sin \theta / \sin \theta,
$$

where c' and c the sound speed in the two media, $\theta$ the angle of incidence and $\theta$ ' the angle of refraction.

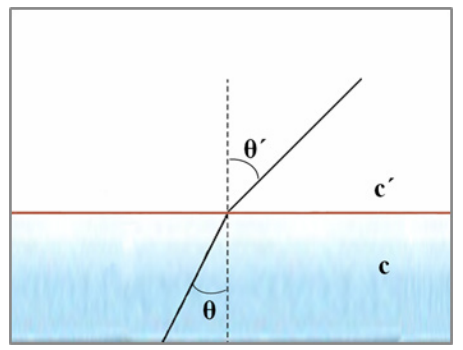

Fig. 3. Refraction - Sound Physical Phenomenon.

\section{BACKGROUND}

The spatial sound rendering has been utilized in many approaches to simulate a realistic aural environment. As a result, a significant number of algorithms have been proposed to develop an innovative solution for this issue [9]. In our research, we used the below classification of these methods in order to survey the sound propagation technique:

\section{Spatial Sound}

- Sound propagation/Audio rendering

- Acoustic Wave Equation Methods

- Finite Element Method

- Boundary element method

- Finite-difference time-domain

- Digital Waveguide Mesh

- Geometric Methods

- Enumerating Propagation Paths

- Image Sources

- Ray Tracing

- Beam Tracing

- Radiosity

- Hybrid Methods

\section{- Web Spatial Sound}

This paper focuses on the most effective auralization techniques and reports both the positives and negatives of each of them. With this in mind, an evaluation of each proposed algorithms/methods is presented. Finally, the fact that despite the significant amount of work that has been carried out with regard to the potential for sound synthesis and sound propagation, there is still a lack of studies regarding the critical issue of spatial sound in web environments is highlighted.

\section{A. Sound Propagation/Audio Rendering}

\section{1) Acoustic Wave Equation Methods}

The most precise propagation algorithms that are intended to simulate several sound effects are based on numerically solving the acoustic wave equation [10]. Specifically, the acoustic wave equation, which describes the physics of sound propagation, is presented by the mathematical equation (2):

$$
\theta^{2} \mathrm{p} / \theta \mathrm{t}^{2}-\mathrm{c}^{2} \nabla^{2} \mathrm{p}=\mathrm{f}(\mathrm{x}, \mathrm{t})
$$

where $\mathrm{p}(\mathrm{x}, \mathrm{t})$ is the acoustic pressure, $\mathrm{c}$ is the speed of sound and $f(x, t)$ a source term describing one or more external sound sources.

Apart from this, the sound propagation has been expressed by the frequency-domain equalization of Helmholtz (3). As it is discussed 
below, there are several methods which use it, in order to estimate the spatial sound in a virtual environment [11].

$$
\nabla^{2} \mathrm{P}+\omega^{2} / \mathrm{c}^{2} \mathrm{P}=0 \text { in } \mathrm{A}^{+}
$$

where $P(x, \omega)$ is the (complex-valued) pressure field, $\omega$ is the angular frequency, and $\mathrm{A}+$ is the acoustic domain.

Taking into account all the above, the ultimate goal of this section is to give an overview of the most well-known numerical techniques, by defining them; listing their advantages and disadvantages; providing examples of studies in which they have been applied. More details on the theoretical basis of the most popular state-of-the-art numerical structural-acoustic methods was presented in "Review of numerical solutions for low-frequency structural-acoustic problems", by Atalla and Bernhard [12]

\section{a) Finite Element Method (FEM)}

Finite element methods (FEM) [13] (volumetric techniques) have as major aim to solve numerically the wave (Helmholtz) equation in boundary conditions. In others words, FEMs try to solve the wave equation through the division of the space into a finite number of small elements (voxels) [14], [15].

Much research on sound propagation using FEM has been done. One of the first studies was developed in 1979 by Dennis W. Quinn [16]. In his work, the FEM was used to compute the sound propagation in non-uniform ducts which contain flow. To verify this approach, it was compared with other solutions or limiting cases and the sample calculations gave satisfactory results, in the case of two dimensional flows within no uniform ducts.

Moreover, a variety of approaches are demonstrated in the review paper examining the use of FEM in acoustic modeling, by Thompson [17]. The review was concluded with the prediction of the continued research on solutions to the challenging problem of developing efficient techniques for acoustic simulation, using finite-element methods, on the ground that it gives satisfactory results when compared to its simplicity.

Additionally, the group of Chou [18] used the FEM to assess the sound field distribution based on the indoor space and chamber volume, taking into account the effects of shape, absorption property, and room boundary, on sound delivery, in order to determine the improvement of interior sound. They reached the conclusion that their proposal can be applicable to the predesign analysis of interior architecture in order to improve the interior noise and reduce the construction costs.

To sum up for this method, it is one of the initial techniques that have been applied for the sound propagation. Additionally, the technique has also been extended in subsequent studies and has been used in hybrid approaches - namely in combination with other methods, in order to overcome some difficulties, as outlined in the literature review. Examples of hybrid methods will be presented in detailed in the corresponding paragraph.

Finally, FEM is not appropriate for open scenes, for the reason that these environments are characterised by sparse scattering geometry and uniform wave propagation speed. For this case, another acoustic wave equation method has been proposed as more suitable technique [19]. It has the similar philosophy as FEM and will be analysed in the next section.

\section{b) Boundary Element Method (BEM)}

The boundary element method (BEM) is a traditional technique which can provide numerical solutions and be applied to a range of engineering and scientific issues.

Particularly, it can be described by the simplicity for the reason that it demands only a mesh of the boundary of the domain. As a result, this technique is more effortless to be used than other classical finite element methods. Hence, the BEM is an integral part in the field of linear acoustics and it has reached a level of maturity in these areas because it can be overcome many of difficulties with low cost and offers adequate solution.

In comparison with the better-known FEM, the BEM differs in the element structure [20]. Specifically, the algorithm can be divided in the follow steps: (1) the Helmholtz equation is transformed into the boundary integral equation; (2) pressure and velocity are solved on the boundary, as a result of that the pressure is calculated at any point in the domain [21].

Overall, it constitutes one of the most accurate propagation algorithms for simulating various acoustic effects, evidenced by the fact that it has attracted much attention from research teams. Indeed it is no coincidence that the first audio representation approaches have been proposed using the BEM. Specifically, the first systematic study on the sound propagation in underwater was carried out by Chen and Schweikert [22], using a boundary element fluid model in combination with a boundary element structural model. The proposed method had the advantage of the readily used in practice, because the theoretical analysis was used in order to a mathematical model be constructed which can be an evaluated tool available for the next studies. Furthermore, Cunefare et al. [23] developed a boundary element method in order to solve the exterior acoustic radiation problem which was produced for the phenomenon of wavenumbers. The strong point of their work was the effectiveness of their method and the overcoming of the major drawbacks using boundary integral methods in acoustic problems (uniqueness of solution, singular integral kernels). Afterwards in 1996, the group of Zhenlin [24] provided a study using BEM to predicting the acoustic performance of expansion chamber mufflers with mean flow and compared it with the corresponding methodologies in the literature with satisfactory results. Moreover, Katz [25] proposed a solution to calculate a portion of the head-related transfer function (HRTF) of an individual based on precise geometrical data based on the BEM. They had found a cutting-edge solution to alter the geometry of the individual through the model in ways which were not possible with real objects. Lastly, Bapat et al. [26] presented a technique in which BEM was adapted in order to model 3-D half-space acoustic wave problems. They concluded with the presentation of results in which they highlighted the decrease of the requirements in the CPU time and memory storage of their algorithm in contrast with previous methods.

\section{c) Finite-Difference Time-Domain (FDTD)}

FDTD has become a commonly used algorithm in room acoustic modeling, for the reason that it is characterized by computational power that is becoming more readily available. It has as its major principle the fact that derivatives in the wave equation are replaced by corresponding finite differences. Furthermore, FDTD has higher precedence, because it produces better suited impulse responses to auralization than FEM and BEM, which typically calculate frequency domain responses.

Due to the above benefits of FDTD, several researchers have proposed interesting approaches for sound propagation using this technique. One of the first examples was presented by Blumricha and Heimannb [27], in which they investigated a simulation of sound propagation in an inhomogeneous atmosphere, avoiding many of the necessary approximations. The contribution of their study was the determination of single atmospheric influences to the whole effect on sound waves. An additional work has been described in the same year (2002) by Salomons group [28]. An interesting approach was analysed for sound propagation in systems with inhomogeneous moving media and finite-impedance surfaces using the FDTD model. Their results have been verified and have been in accordance with the solutions of the 
Helmholtz equation. Furthermore, Heutschi et al. [29] based on FDTD in order to capture the typical ground impedances in the low-frequency range for outdoor soils adding small computational cost. Extensive results carried out showed that this method had good agreement in comparison with analytical solutions. Lastly, in [30] an adapted FDTD model was presented to be applied to a terrain-following coordinate system taking to account the orography of the ground surface. The results demonstrated in this study match state of the art methods.

However, the complexity of numerical methods increases linearly function according to the surface area of the primitives or the volume of the acoustic space, and as at least a cubic function of the maximum simulated frequency. Recently, many wave-based pre-computation techniques have been proposed for interactive applications. The reason being is to take advantage of numerical methods but not use it in real time, which increases the computational cost.

\section{d) Digital Waveguide Mesh (DWM)}

DWM approaches are numerical simulation techniques which use discrete waveguide elements; each of this element carries waves along its length in single dimension. One of the first systematic analysis was described by Duyne and Smith [31] in which an adequate technique was developed extending the DWM to model the propagation of the wave in a membrane. Additionally, Savioja group [32] presented a detailed analysis of DWM and proposed some methods in order to overcome its major drawback which is the direction dependent dispersion. Also, Murphy [33] developed an innovative environment which offered for research into the application of DWM-based models for virtual acoustic spaces.

However, DWM methods suffer from directional dispersion of sound, that is, sound does not travel with the same speed in different directions on the spatial grid [34]. Due to the fact that DWM also had significant drawbacks, the following research should use more sophisticated techniques to overcome the limitations in numerical methods and produce a realistic sound simulation.

\section{2) Geometric Methods}

The main benefit of the acoustic wave equation methods is to yield accurate results for the physical propagation of sound, by solving numerically the wave equation. However, they also exhibit a number of drawbacks. Specifically, their complexity depends both on the simulation frequency and on the surface areas of the objects or the volume of the acoustic space [35]. So, these methods are sensitive to the complexity of the virtual environment, which means that they are mainly limited to realistic scenes. To put it differently, these techniques are computationally too expensive for dealing with the whole audible frequency range and they are inadequate for high frequency auralization. As a result, these techniques are not appropriate for interactive sound propagation in complex environments. [19], [36-42].

For this reason, geometric methods were used in order to overcome some of the above disadvantages of wave methods, so that the $3 \mathrm{D}$ sound can be embedded into more sophisticated and complicated applications.

Firstly, from a theoretical point of view, sound and light are both waves; as a result, they share many common properties and similar techniques can be used in order to be rendered in a virtual scene. With this in mind, many algorithms which have been used for light rendering, they were adapted to solve the sound rendering in a 3D scene.

However, the extensive analysis of these two phenomena identifies some significant differences between them. Firstly, from the physical point of view, sound waves cover a much broader range of different wavelengths (the wavelengths of audible sound fall between 0.02 and 17 meters and their correspondent frequencies are $20 \mathrm{kHz}$ to $20 \mathrm{~Hz}$ ), more than three orders of magnitude larger than visible light. Moreover, as a result of the long sound wavelengths in comparison with the dimensions of most common objects in a room, the sound modeling requires less detail of room geometry. In general, the most common behavior of sound waves reflection in large objects (such as walls) is the specular. On the other hand, significant diffraction is occurred in the around edges of objects. Small objects have meaningful effect on the sound propagation only in the case that frequencies are over 4 $\mathrm{kHz}$, so they can usually be excluded from auralization algorithms, especially in the presence of other sources with considerable reflection and diffraction phenomena. Secondly, sound travels through air roughly 106 times slower than light. As a result, the acoustic signal is perceived as a combination of direct and reflected sound, because its speed causes obviously different arrival times for sound propagating in different paths. Lastly, since sound is a coherent wave phenomenon, the computation of the reflected and scattered sound waves should incorporate the phase (complex amplitude) of the incident and reflected waves. In contrast, the incoherent light demands only the sum of the power [43].

Despite the above differences between sound and light, the algorithms of sound propagation borrow the most of the light rendering algorithms and techniques.

a) Enumerating Propagation Paths

\section{Image Sources}

The main principle of the image method is to enumerate specular reflection paths, by taking into account virtual sources. The virtual sources are constructed like mirroring location of the initial source, with respect to all polygonal surfaces of the environment. In other words, a sound source is reflected against all surfaces in a model; as a result, a set of image sources is produced. These are again reflected against all the surfaces by an iterative process, until a termination condition is satisfied. Response length or reflection order could be examples of these conditions in order to finish the algorithm and return the result [44].

Additionally, a hierarchical image-source tree can be used in order to depict the result of the image-source computation. In this structure, the root is the sound source and each branch represents an imagesource. Consequently, an impulse response for acoustic scene can be calculated as the sum of all image-sources, which are included in the environment and they are the branches of that tree. Also, from the computational point of view, this method is used for the computation of the first reflections to avoid the significant increase of cost which depends directly on the number of image sources. [45].

A growing body of literature has examined and analyzed the imagesource algorithm in the field of sound propagation. The interest in this area is demonstrated by the increasing number of reviews and the systematic study on this topic by other researchers [3], [9], [7], [44].

In an early study, Gibbs and Jones [46] used the image source method in order to measure the variation of sound pressure using a constructed rectangular model which possess a significant number of absorption configurations. Later, Santon [47] presented an approach for the estimation of speech intelligibility in rooms, using the imagesource technique. His proposal takes into account the directional distribution of the echoes and is based on the concept of received energy being partitioned into useful and disturbing energies. This method was evaluated and was characterised as a clear improvement over the other techniques for predicting speech intelligibilities. Another study by Allen and Berkley [48] developed the impulse response between two points in a small rectangular room. This work was characterized by simplicity and was implemented for a room with rigid walls with only specular reflections and no diffractions. In 1984, Borish [49] improved the image-source method described by Santon [47], in order to extend for arbitrary polyhedra with any 
number of sides. Later, Heewon Lee and Byung-Ho Lee [50] presented an algorithm for the simulation of sound ray paths in an arbitrary polyhedral room, which is based on the image model algorithm. After evaluations, they concluded that their method improved the efficiency of the image model technique by compensating for the drawbacks of corresponding methods. Furthermore, Vorlander [51] proposed a new method which combined both image-source model and the ray tracing. In 1992, Renate Heinz [52] developed an innovative approach in which they extended the image source method with a diffuse background signal to its result. In this study, only the specular parts of the reflections were considered and analysed. The following decade, Lehmann and Johansson [53] introduced a new method which provided an approximation of the acoustic energy decay (energy-time curve) in room impulse responses generated using the image-source technique. This study gave the advantage to researchers to undertake a preliminary analysis of a simulated reverberant scene without the need for time-consuming image method simulations. One year later, McGovern [54] addressed two major disadvantages of the image source method (redundant or unnecessary mathematical operations). In the first case (redundant mathematical operations), the use of look-up tables was proposed and in the second case (unnecessary mathematical operations), he used a sorting method. The evaluation implied that this method resulted in a substantially reduced computation time, and thus had potential applications for real-time auralization in interactive scenes. In addition, in 2013, André's group [55] analyzed the issue of auralisation which was based on geometric acoustic models. Specifically, the direct sound and reflections from each source were computed dynamically by the image-source method with the combination with HRTFs. Subsequently, trying to improve image methods for real time approaches, Charalampous and Michael [56] implemented an image source method variant in which they compared three different tree traversal approaches, depth-first, breadth-first and best-first.

As reported in the above studies, the fundamental benefit of these methods is their robustness. Specifically, they offer the security that all specular paths up to a given order or reverberation time will be found. On the other hand, the computational complexity of these methods grows exponentially, despite the fact that they compute only specular reflection. A solution to this problem has been achieved through the introduction of a ray tracing algorithm, which is analyzed in the next section.

\section{Ray Tracing}

The concept of this method is to compute propagation paths of sound that arrive to a receiver by generating rays emanating from the source and following them through the environment until an adequate number of rays has been found that reach a receiver position. [9], [7]. To put it another way, during the first phase the sound rays are produced in all directions. These rays are reflected at surfaces and the aim of this step is to find which of them hit any listener because this means that the specific ray is audible. It is obvious that the sound propagation has a number of similarities with the problem of solving global illumination by ray tracing, which is used in light rendering, and a similar method can be applied in the sound field.

According to the way sound sources emit rays and depending on how these rays interact with objects of the environment, different versions of the ray tracing algorithm have been developed. This means that these methods take into account the directions of rays (fixed/random) or the absorption of the incoming wave by the surfaces. Specifically, the size of the absorption of any surface should be identified from a coefficient which depends on the wavelength of the incoming sound. Finally, there are many ray tracing methods, which can also handle diffuse reflections and take into account the percentage of diffusion or scattering, but in these cases the computation time significantly increases [57].

There is a considerable amount of literature on the issue of sound propagation using the ray tracing technique, a fact that demonstrates the significance of the specific algorithm.

The first systematic study on the distribution of early reflected sound over the audience areas in concert halls was carried out in 1968 by Krokstad using a ray tracing technique [58]. Afterwards, Kulowski in 1982 [59] indicated a method of determining a quantitative measure of the ray tracing technique error. The importance of this study lies in the fact that it offered the option to enumerate sound decay curves of comparative credibilities, which is especially useful when curves are being modeled in different observation regions or even in different rooms. Two years later, the same author presented an algorithm which was based on the ray tracing method. This proposal gave the opportunity to model the acoustical field in rooms using small computers, because the calculation time was decreased sufficiently, a fact which has been confirmed using several examples [60]. Furthermore, in 1993, Lehnert [61] not only analyzed the two main kinds of inherent systematic errors of this method (errors due to a detection problem and errors due to limited spatial resolution), but also proposed an algorithm for this purpose which was validated perceptually. In the same year (1993), van Maercke and Martin [62] designed an approach to artificial reverberation in acoustics, without taking to account the diffuse in the environment of simulation. It contained a ray tracing algorithm for the calculation of echograms and implemented a beam method to predict short time impulse responses and criteria maps. Additionally, Li, Taherzadeh and Attenborough [63] used and extended the previous ray tracing scheme, in order to predict the sound field near a flat impedance ground in a refracting atmosphere that includes the effect of vector wind and turbulence explicitly. Before that, Mueller and Ullmann [64] described an approach to enhance sound by high quality $3 \mathrm{D}$ audio information through acoustic ray tracing. The main objective of this work was to compute a fixed sound source for a constant listener, with the prospect of an extension of the method in which 3D audio for moving listeners could be generated in interactive environments. Furthermore, Alpkocak and Sis [65] presented an approach to calculate the impulse response of a room using the ray tracing algorithm. This is based on assumptions that the environment was linear time-invariant system and the impulse response was calculated by sending Dirac impulses into the system as input and then the output gave the response. Moreover, ray tracing was attractive for further study. For example, the group of Dreher [66] tried to study the 3D ray tracing algorithm in the environmental noise context. The major goal of this approach was to reduce the computation time using different acceleration structures. Other work from the group of Okada [67] was presented in which novel ray tracing method was developed in order to solve sound diffraction problems using the ray tracing due to calculate sample values of the integrand. Besides, the evidence from this study intimated that this method offered applicability, after its evaluation with a prototype system of interactive ray tracing.

Furthermore, in the last few years, considerable attention has been paid to sound propagation for more sophisticated and realistic implementations. Specifically, Taylor et al. [68] introduced an attractive method for tuning geometric acoustic simulations based on ray tracing. They demonstrated that their technique achieved a significant performance improvement over prior geometric acoustic methods for the same number of contributions. As a result, the system had the ability to render acoustic spaces composed of thousands of triangles interactively. Also, Mo et al. [69] developed an efficient algorithm which is based on ray tracing, in order to simulate sound propagation in large outdoor scenes. The peculiarity of these environments was the variety of objects and the complexity of objects' boundaries. After that, the same group presented a paper in which the ray tracing was 
used in order to improve the efficiency of outdoor sound propagation, without significant limitations of the scene, by the utilizing of analytic ray curves as tracing primitives [70]. In the same way, in one of the most recent publications [71], Schissler and Manocha computed the propagation paths from each source to the listener in large, dynamic scenes using ray tracing. Through this approach, they succeeded in accelerating the computation of impulse responses for interactive sound rendering in a $3 \mathrm{D}$ virtual environment.

As mentioned in the literature review, one of the essential advantages of this method is the simplicity. Furthermore, the speed and the efficiency on GPU hardware are additional strong points of the ray tracing algorithm. Finally, it offered the possibility to consider both higher order reflections, without significant computation cost increase and diffuse reflections. On the other hand, ray tracing is a stochastic method and one of its drawbacks are sampling artifacts as well as possibly lost important sound paths due to the limited sampling steps. There is no one guarantee that all significant paths will be considered. In addition, diffraction is theoretically possible but cannot be solved efficiently. This appears because the more distant a ray has traveled, the more sampling artifacts will occur. As a result, many rays are computed that will never reach the listener [7], [72].

The evidence from two algorithms analysis (image sourceray tracing) for sound propagation intimates that they have both advantages and disadvantages. Therefore it is worthwhile developing a combination of both in order to obtain fine temporal resolution in sampling rate quality, taking to account the phenomenon of scattering and faster audibility check of image sources [8]. This combination is called a "hybrid method" with key advantage of this approach being that a number of weak points in one algorithm can be ameliorated using strengths of the other. More details on this are given below.

\section{Beam Tracing}

This methodology was firstly developed in computer graphics in order to utilize the spatial coherence in generating realistic images and after that it was adapted for the sound rendering. In the sound propagation case, it classifies the propagation paths from a sound source, using the recursive method to trace the pyramidal beams (i.e., sets of rays) through the acoustic scene Particularly, for each beam, polygons in the scene are considered for intersection with the beam in front-to-back visibility order. During the algorithm firstly, polygons are detected, secondly, the original beam is clipped to delete the shadow region, thirdly, a transmission beam is constructed to match the shadow region, fourthly, a reflection beam is produced by mirroring the transmission beam over the polygon's plane, and finally conceivably other beams are created in order to model other types of scattering (see Fig. 4) [9], [72].

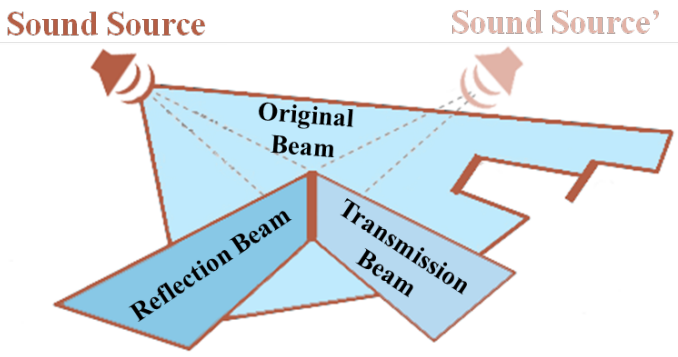

Fig. 4. Beam Tracing Method.

To review, there is a considerable amount of literature on this algorithm and especially if it is combined with another method of sound propagation. Preliminary work was carried out in the early 1990s, by Lewers [73] in which he proposed a beam tracing model in order to predict the behavior of sound in a room. Then, Funkhouser's group [74] developed new beam tracing algorithms that greatly accelerate computation of reverberation paths in a distributed virtual environment by taking advantage of the fact that sounds can only be generated or heard at the positions of listeners, which were represented by avatars. In this work, they succeeded in developing a faster beam tracing sound propagation technique which could support real-time computing.

Additionally, Funkhouser et al. [75] describe a beam tracing method based on precomputed spatial subdivision and "beam tree" data structures that enables real-time simulation of sound for static sound sources in interactive virtual environments. The offered advantages of this technique, in contradiction with earlier literature, were the scale, the accuracy, and the interactivity.

Moreover, others have analyzed a new theory which extended geometrical acoustics with diffraction phenomena [76]. The strength of their contribution lies in the fact that it is a new beam tracing method for enumerating sequences of diffracting edges efficiently and without aliasing in densely occluded polyhedral environments; it resulted in a practical approximation to the simulated sound field in which diffraction was considered only in shadow regions; additionally, it resulted in a real-time auralization system demonstrating that diffraction dramatically improved the quality of spatialized sound in virtual environments.

Further important research was proposed by Funkhouser's group [77]. Specifically, in this work, a beam tracing method was developed in order to enable interactive updates of propagation paths from a static source to a moving listener in large indoor area. Firstly, the major advantage of this approach is the ability to support auralization in large building environments. Secondly, it simulates sound propagation due to edge diffraction. Third, it finds all propagation paths up to a given termination criterion without exhaustive search or risk of undersampling and finally it updates propagation paths at interactive rates.

Furthermore, Ajaj, Savioja and Jacquemin [78] developed an innovative environment for the purpose of the sound propagation in an interactive virtual scene. In their development, they based their approach on the beam tracing algorithm in order to implement the one part of this application for a real-time acoustic simulation. Also, the group of Laine [79] proposed an advanced solution which improved the previous method of beam tracing, in order to accomplish an optimized algorithm for finding and efficiently updating specular reflection paths for a moving listener. They demonstrate that the proposed algorithm performs well both with complex, lightly occluded room models and with moving sound source at interactive rates with moderate model complexity.

Furthermore, Antonacci, Sarti and Tubaro, in 2008, proposed a novel technique that could enable the fast tracing of a large amount of acoustic beams through the iterative lookup of a special data structure that could represent the global visibility between reflectors. After two years, this group analyzed an extension of the previous work in which diffraction and diffusion were simulated in the model to succeed more realistic results, without considerably increasing of the computational efficiency. This expanded approach demonstrated that not just the construction of the beam-tree but also the whole path-tracing process can be performed entirely on the visibility maps [80], [81].

As others have highlighted, beam tracing is currently considered to be the fastest commonly used geometric room acoustics modeling technique [72], [79], [82], [83]. This algorithm has advantages over both image source and ray tracing. Comparing with the image source method, the beam tracing advances in the fact that fewer virtual sources must be considered for the sound propagation algorithms, which is an important factor of effectiveness in geometrical complex scenes. Since each beam represents the region of space for which a corresponding 
virtual source (at the apex of the beam) is visible, higher-order virtual sources must be considered only for reflections of polygons intersecting the beam [9]. On the other hand, in contrast to ray tracing, beam tracing works with object-precision polyhedral volumes that support welldefined intersections with diffracting edges. As a result, beam tracing is not affected by aliasing phenomena [76], [84], [85]. Additionally, it advances in the geometric coherence, because the algorithm calculates for each beam an infinite number of potential ray paths from the source to the listener. As a result, beam tracing is independent of the sampling artifacts, such as the ray tracing. [77].

Finally, Charalampous and Michael in their review paper [72], underline that beam tracing transcends as a deterministic method, in comparison to ray tracing. At this point, it should be noted that deterministic method will produce the same results when run multiple times. For example, a deterministic algorithm for detecting sound reflections, in a specific model, will detect the exact same reflection paths up to a given order of termination each time executed.

In contradiction with the above analysis of beam tracing benefits, this method is characterized from drawbacks too. For example, the geometric operations required to trace beams through a 3D model, such as intersection and clipping, are relatively complex for the reason that there is the possibility for each beam to be reflected and/or obstructed by several surfaces [77], [9]. Likewise, the group of Funkhouser [74] at the Bell Laboratories point out another disadvantage of the beam tracing, in that they notice the fact that the particular algorithm is difficult in environments with curved surfaces and non-linear refracting objects. They propose, as suitable solutions in these cases, the conservative beam tracing methods combined with validation of constructed paths.

\section{b) Radiosity}

Some preliminary work for the radiosity method was carried out in the 1950s, in the field of thermodynamics. Although, the basic equations of this technique have been presented in an optics paper by the Yamauti [86] and have been attractive for computer graphics since 1980s. Without delay, this technique was suggested and developed in acoustics, for the reason that both light and sound share many similar properties. On the other hand, there are some principal differences between radiosity in acoustics and radiosity in computer graphics, such as time-dependence. Particularly, sound is in contradiction with light, because it travels so slowly through the air. This fact causes a significant time delay which cannot be disregarded by any model of sound propagation. As mentioned below, it is one of the limiting aspects of acoustical radiosity because of the high computational cost [87].

There is a considerable amount of literature on acoustic radiosity. Some preliminary work was carried out several years ago. In 1993, the group of Shi [88] proposed a modified radiosity algorithm in order to implement both visual and room auditory rendering. After a few years, Tsingos and Gascuel [89], [90] presented new approaches for the simulation of room acoustics based on hierarchical radiosity. These are novel approaches because complex phenomena, such as sound global specular and diffuse reflections, were taken into account for the first time, in order to make their methods promising for virtual acoustics applications. Afterwards, at the beginning of the next decade, Nosal and co-workers [91], [92] developed a radiosity algorithm for rectangular rooms while they focused on addressing the problem of the applicability of the method in the light of the inherent assumption of diffuse reflection. Furthermore, in 2004, a mini review of radiosity method in simulating sound fields with diffusely reflecting boundaries was presented by Kang [93]. Additionally, Nosal et al. extended their work ([91], [92]) documented in another paper in which they investigated how acoustical radiosity performs in predicting real room sound fields (a squash court, a classroom, and an office). Moreover, Siltanen et al. [94] proposed a new extended acoustic radiosity method to solve the room acoustic rendering equation in order to handle both diffuse and non-diffuse reflections. Finally, different from all the previous literature, Muehleisen [95] suggested the radiosity for the prediction of sound pressure levels in six sided rectangular rooms.

As reported previously (in Section 2.1.2.2), the sound radiosity is limited by the fact that it is time dependent, in comparison with the corresponding method in light. Nevertheless, this feature can be an advantage on the ground that this computational cost is incurred only in the initial sound rendering. Specifically, the estimation for the sound rendering in an area will need to be done once at the beginning for a given source. After that, the remaining computational costs are adequate low to enable real-time sound simulation for moving listeners. In addition to this, several approaches have been proposed to accelerate the initial rendering, in order to improve this issue [92].

\section{3) Hybrid Method}

As outlined in the above literature review, classical methods for sound propagation are typically limited for realistic results for a number of practical interactive applications. Particularly, wave-based methods are adapted to the lower frequencies and relatively small domains but are not sensitive to the complexity of the domain. On the other hand, geometric methods are dependent on the number of successive reflections in the domain, and thus to their complexity [96]. For this reason, a number of hybrid methods have been developed and suggested by combining the classical sound propagation methods. This model was chosen because it is one of the most rapid ways to take the advantages of the above methods, in order to generate realistic sound effects, including reflections, reverberations and succeed significantly reduced calculation times.

The significance of the hybrid method is confirmed by the more recent literature. One of the first studies in this field, Tsingos et al. [76] extended the beam tracing algorithm to construct propagation paths with diffraction, and they introduced a practical approximation to the diffracted field in shadow regions. Their evaluation clearly established that (1) beam tracing algorithm is an efficient and unaffected from aliasing phenomena to compute diffraction sequences in densely occluded acoustic scenes, (2) it is an effectively technique to produce early diffracting propagation paths and auralization in real-time and (3) diffraction greatly improves the quality of spatialized sounds in immersive virtual environments. After that, Sikora [97] reported a hybrid algorithm using beam tracing method, which was intended to solve the problem of the refraction, without sacrificing the accuracy and efficiency of beam tracing method. Furthermore, Tsingos et al. [15] presented an auralization framework which gave the opportunity to render scattering effects interactively thus providing a more compelling experience, based on the programmable graphics hardware for all geometric computations. Also, after validation examples, their results showed for the first time that the Kirchhoff approximation can be successfully used for off-line sound propagation in very complex scenes. Also, Lauterbach, Chandak and Manocha [5] combined two different methods in order to produce a innovative algorithm for real-time auralization in complex, dynamic virtual environments. Particularly, they used the ray tracing which gives realistic acoustic simulation in interactive environments and the frustum tracing for the volumetric representation.

Moreover, Stavrakis, Tsingos and Calamia [98] presented a novel graph-based topological sound propagation algorithm that can compute interactive reverberation effects in complex coupled environments, in which they used auditory masking and scalable Fourier domain processing to render a large number of reverberated components. Their work clearly had some limitations, but it was a springboard for 
the development of interesting real-time sound propagation methods and it could find applications both for acoustical design and virtual environments. In the same token, the group of Taylor [99] presented an interactive algorithm which combines both geometric propagation techniques to compute the propagation paths and a ray-based underlying representation that is used to compute specular/diffuse reflections and edge diffraction. Similarly, Pohl and Stephenson [100] described a new idea of combining ray tracing with the radiosity method to a very efficient geometric simulation method including diffraction and scattering. After the evaluation of the proposed hybrid algorithm, they reached the conclusion that the computation time was reduced from exponential to linear growth with split-up of sound particles, but the method became ineffective in case of no split-up.

Additionally, Yeh et al. [4] presented a novel hybrid approach that combines both geometric and numerical acoustic techniques for interactive sound propagation in complex environments. Specifically, they used wave-based techniques to pre-compute the pressure field in the near-object regions and geometric propagation techniques in the far-field regions to simulate the sound propagation in a scene. They demonstrated that their system was able to simulate high-fidelity acoustic effects such as diffraction, scattering, low-pass filtering behind obstruction, reverberation, and high-order reflections in large, complex indoor and outdoor environments with a satisfactory realistic result. Also, the pressure computation requires orders of magnitude lower memory than standard wave-based numerical techniques. Moreover, Pelzer, Masiero, Vorländer [101] proposed a hybrid reproduction approach, in order to succeed a realistic and natural sounding high quality auralization of sound sources in enclosures, by using binaural technology including near-field effects for close sources and employment of individual head-related transfer functions. In the same year, 2014, the group of Schissle [10] developed a hybrid algorithm with the merger of radiosity and path tracing techniques. They managed to address the problem of interactive sound propagation and rendering in large-scale virtual environments which are composed of multiple moving sources and objects. They demonstrated an order of magnitude performance improvement over previous methods, through the performance of their method in complex indoor and outdoor environments.

Furthermore, in [102] a novel algorithm was recommended to accurately solve the wave equation for dynamic sources and listeners using a combination of pre-computation techniques and GPU-based runtime evaluation. It was proved a significant improvement in runtime memory comparing with prior wave-based techniques which were applied to large scenes with moving sources. Likewise, Charalampous and Michael [103] introduced a hybrid sound propagation algorithm in which image source method was used to calculate sound reflections from specular surfaces and a prioritized ray tracing algorithm for fast detection and evaluation of valid image sources from the tree of candidate image sources. After the comparison with other algorithms, such as ray tracing algorithm and best first image source algorithms, they demonstrated that acoustical results were improved in most cases. A further important publication is from Podkosova group [104] which developed a hybrid sound model based on the image source method and the secondary sound sources for late reflections and reverberation. The motivation of this approach is that a complex real-time hybrid model enhances task performance in 3D audio games when compared to a basic model. In addition to the previous work, Rungta et al. [105] developed a coupled sound synthesis-propagation algorithm that can generate realistic sound effects for computer games and virtual reality, by combining modal sound synthesis, sound radiation, and sound propagation. In order to perform the sound propagation, they used the fast ray tracing technique to compute the impulse responses using perceptual Hankel approximation. Add to this, with the use of
3D virtual complex indoor and outdoor scenes, they confirmed that the proposed method can handle a high degree of dynamism in term of source radiation and propagation in complex scenes.

Finally, Schissler and Manocha [35] from the University of North Carolina at Chapel Hill presented an interactive algorithm for sound propagation and rendering in complex, dynamic scenes with a large number of sources, which combined fast backward ray tracing from the listener with sound source clustering to compute propagation paths. They demonstrate their algorithm's performance on complex indoor and outdoor scenes with high acoustic complexity and observe significant speedups over prior algorithms.

\section{B. Web 3D Spatial Sound}

Many approaches for Web 3D applications aimed at realistic visualization of the scene. As in the above desktop embodiments so in web applications, spatial sound can offer further details to a 3D graphic world.

As a result, the first attempt took place with the use of the $<$ bgsound $>$ tag, in which only background music could be contained in a web page and was not being supported from all browsers. After that, flash was the first cross-browser way of audio on the Web, but a key limitation of this research was the requirement of the plugins installation. Moreover, the focus of following research was concentrated on the element < audio> in HTML5, which could avoid the plugins, but was not designed for sophisticated and complex applications [106], [107]. Particularly, the element < audio $>$ is inferior to apply filters to the sound signal and access the raw PCM data. Furthermore, the orientation both of listener and sound source is not available; as a result the proposed method cannot be readily used in practice. Lastly, it does not afford low-latency precise-timing model, which is very important in order to develop interactive applications, with fast auditory response to user actions [108]. Thus, it is not adequate for a 3D interactive web scene with demanding sound design.

Under these circumstances, several alternatives have been proposed, in order to establish an effective API, which attends to overcome the most of these limitations. One of the most interesting approaches to this issue is Web Audio API, which has been proposed by Mozilla Foundation.

Indeed, it was not a coincidence that Web Audio API has gained much attention from researchers in the last years. In particular, the literature demonstrates a variety of studies which utilize Web Audio API, in order to accomplish the sound in browser. For instance, Choi and Berger [109] developed a JavaScript library which is based on the Web Audio API in order to facilitate music in the web environment, bypassing underlying tasks and augmenting useful features. Additionally [110], an innovator framework was described which used the Web Audio API to render object-based 3D audio in a web browser without requiring plugins. Similarly, Rawlinson et al. [111] have also presented an audio feature extractor library using Web Audio API. Their study was a lightweight implementation which was characterized by flexibility and adaptability to introduce audio in a web system. Furthermore, a similar work was introduced by Kleimola and Larkin [112], which included audio effects for web browsers and built on top of Web Audio API. The strong point of this work was the directly loading from the open web without manual installations. Moreover, Pendharkar et al. [113] proposed a new engine which was ported from Adobe's Flash platform to Web Audio API. The main objective of this study was to overcome the differences of the architecture between Adobe's Flash and Web Audio API (the most well-known techniques for web auralization in literature). As a result, many applications had not to be redesigned to work both of them. Another approach, which based on the Web Audio API, was analyzed by Schnell group [114]. This module analyzed a novel solution for the synchronization, schedule and aligning of the 
audio playback in the internet environments. Finally, Mahadevan et al. [115] described a novel learning environment which offered a web platform for teaching computer science through algorithmic music composition.

This fact seems to be justified because Web Audio API is open source and be supported from the most browsers. Except that, it offers multi-channel audio and high-level sound abilities as filters, delay lines, amplifiers, spatial effects (such as panning). Also, audio channels can have 3D distribution according to the position, speed or direction of the viewer and the sound source.

Different from the above publications, the first systematic study for the implementation of spatial sound in web presented from the group of Stamoulias [116] in which they enriched the X3DOM framework (an open source JavaScript framework, used to create declarative 3D scenes in Web pages) with spatial sound features, using both the X3D (a royalty-free ISO standard XML-based file format for representing 3D computer graphics in web) sound nodes and the structure of Web Audio API.

On the other hand, due to the continuous interest of high degree of realistic in web 3D environments, neither the Web Audio API nor any other approach enrichment them with immersive sound characteristics. Particularly, characteristics such as surface reflection, reverberation, physical phenomena including interference and diffraction have not yet been included in web 3D scenes, even though they play a major role in the representation of a realistic $3 \mathrm{D}$ sound. Consequently, it is understandable that there has been little discussion on interactive web 3D immersive environment with the addition of spatial sound effects by taking into account the geometry of the scene.

\section{SUMMARY AND OUTLOOK}

This review has provided a summary of the most significant research taking place in the field of the spatial sound propagation. Specifically, we tried to present the techniques and the algorithms which have been designed in order to provide spatial sound for dynamic and interactive environments, both for the sound propagation and the audio rendering.

After this study, we observed that there is a vast amount of literature on the auralization area [117]. However, this is not particularly surprising given the fact that audio technology has reached a point where algorithms, hardware, and auditory display technology are becoming standard components and be applicable in many fields (video games development, virtual reality, acoustics engineering and other disciplines).

A further conclusion that emerges from this research is that most of the researchers proposed geometric sound propagation algorithms in order to succeed realistic auralization in large scenes with a high number of objects. This fact is justified on the grounds that geometric techniques can be used for fast computation of propagation paths from a source to a listener and takes into account the most of the physical sound characteristics such as specular reflections, diffuse reflections and edge diffraction. In practice, this approach can give a realistic impression of a dynamic sound environment in real time.

Except the geometric proposed algorithms, a growing body of literature has proposed hybrid methods for the sound propagation, as effective techniques. A satisfactory explanation for this outcome can be the fact that this method combines different algorithms and thus takes the advantages of all of them. For the same reason, hybrid method can overcome the most of the major drawbacks that should be addressed during the spatial sound synthesis.

To give an illustration of the results which are generated by this research, we summarize the principal advantages and features of each sound propagation algorithm, in Table I. The strong point of this table lies in the fact we recommend the method that best suits in any case of acoustic approaches, taking to account a significant body of literature.

Namely, Table I compares the main categories of sound propagation methods and indicates the advantages/disadvantages each of them. Initially, it presents the Acoustic Wave Equation Methods - FEM, BEM, FDTD - which are the most prominent numerical techniques for solving the wave equation. In general these methods are widely used for the reason that they can accurately simulate all acoustic effects with low computational complexity. Specifically, FEM and BEM have traditionally been employed mainly for the steady-state frequency domain response, as opposed to a full time domain solution of the wave equation. As a result, FEM is suitable for the interior and BEM for the exterior scattering problems. The FDTD algorithm, on the other hand, produces better suited impulse responses to auralization than the above methods and it has become a commonly used algorithm in room acoustic modeling. However, the requirements of Acoustic Wave Equation Methods increase significantly for complex scenes, so under those circumstances, they are not recommended to model the sound in interactive virtual environments and are perceived as too slow for real time sound rendering.

Furthermore, the next group of sound rendering algorithms, which is described in Table I, is the category of Geometric Methods. Particularly, the first mentioned method - Image Sources - is a technique that provides accurate results, as it detects all the possible sound reflections in a dynamic scene, as a result it is suitable for sound propagation in indoor and outdoor spaces with geometrically reflecting boundaries and without aliasing issues. Additionally, Ray Tracing is the second geometric method. This algorithm is of widespread interest due to the fact that it handles dynamic scenes, taking to account demanding physical characteristics (such as diffuse reflection) with simplicity and generality. In the same fashion, beam tracing method belongs in the same sound propagation category. It benefits from the fact that it is the fastest commonly used geometric room acoustics modeling technique and can handle moving listener. Also, in comparison with other methods, like ray tracing, beam tracing clearly has an advantage as a deterministic method. Finally, the radiosity is suitable for the simulation of sound propagation in urban auditory environments and predicts room sound fields with some accuracy. Despite the fact that the Geometric Methods have many advantages over Acoustic Wave Equation Methods, they have failed to be applicable to interactive reverberation effects and not to be susceptible to aliasing errors. So, taken together, Geometric Methods are distinguished by the benefits of effectiveness, speed and the efficiency for the computation of complex physical sound phenomena, such as reflections and diffractions. Despite the fact that they have many advantages over Acoustic Wave Equation Methods, they have failed to be applicable to interactive reverberation effects and not to be susceptible to aliasing errors.

The last sound propagation category which is highlighted in Table I, the Hybrid Methods, has been suggested in order to overcome the limitations of the previous methods. In particular, these algorithms can be readily used in practice for realistic sound effects, including reflections, reverberations and succeed significantly reduced calculation times; also, they solved the problem of the refraction in dispersive environment. As a result, they can be used in computer games and virtual reality to generate realistic sound effects.

As was mentioned, more details on this issue are given in Table I.

In conclusion, it is evident from this study that the field of the spatial sound propagation is still required research and development. There has also been much progress in the auralization during the past decade, but the real challenge is the sound modeling in dynamic environments in which we can interactively change everything by including the geometry and materials in real time. 
TABLE I. Units For MAgnetic Properties

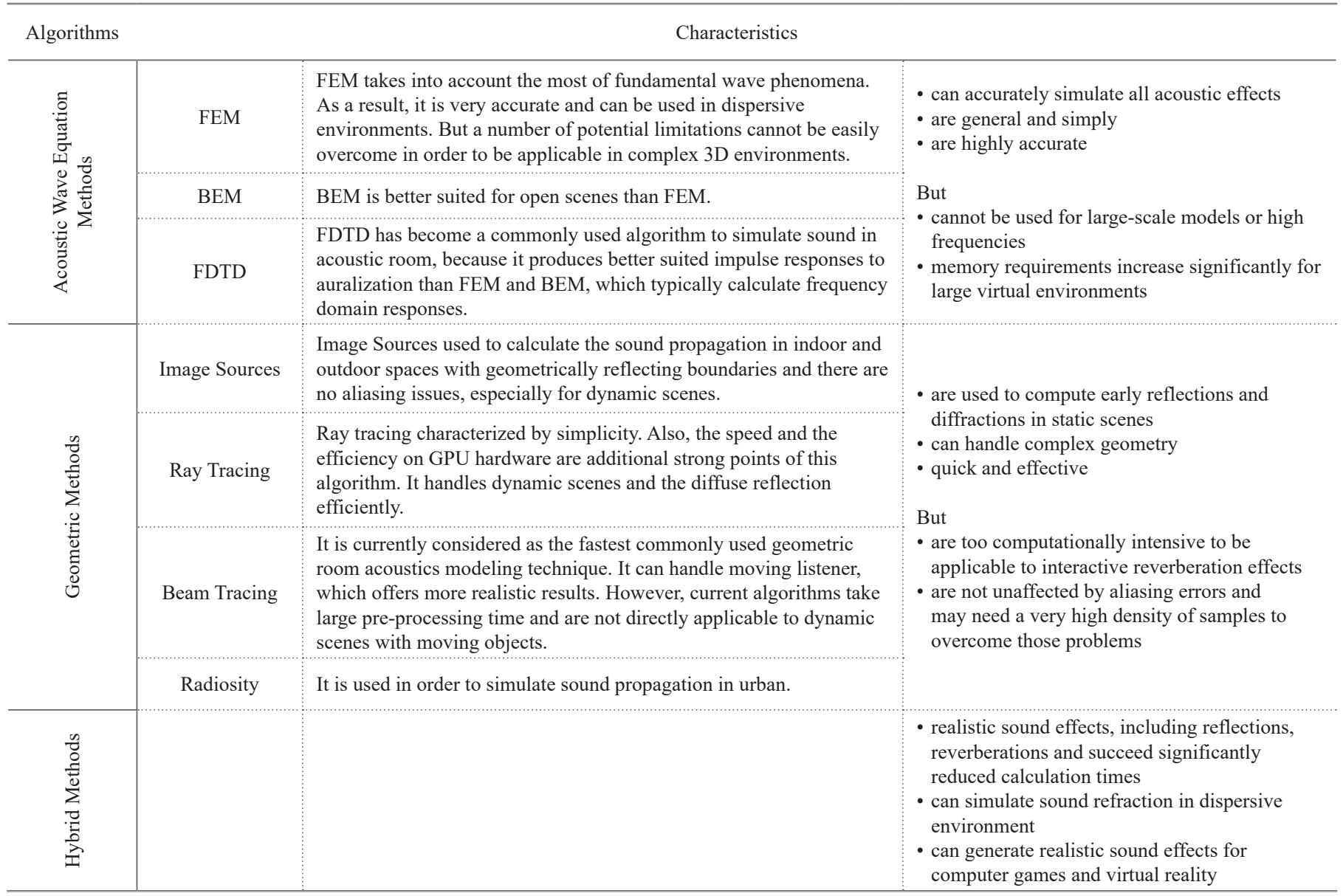

Further work needs to be done in the development of sound propagation algorithms which are based on geometric acoustics and take into account physical phenomena in a web 3D virtual scene. Previous research can only be considered a first step to include not only spatial information, but also physical characteristics of the sound propagation in a browser. To fill this literature gap, the focus of recent research should be on synthesizing and processing high quality audio in web environments. In other words, many acoustic effects including surface reflection, reverberation, physical phenomena such as interference and diffraction, the absorption, coefficient of materials should be taken into account, in order to increase the realism of the sound in a web $3 \mathrm{D}$ environment. This may be considered a promising aspect of the auralization to expand the field of immersive sound beyond the limits of the current web 3D technology.

\section{REFERENCES}

[1] M. Naef, O. Staadt and M. Gross, "Spatialized audio rendering for immersive virtual environments", VRST " 02 Proceedings of the ACM symposium on Virtual Reality Software and Technology, pp. 65-72, 2002.

[2] M. Pallot, P. Daras, S. Richir and E. Loup-Escande, "3D-live: live interactions through 3D visual environments", Proceedings of the 2012 Virtual Reality International Conference, article No. 23, pp 82012.

[3] F. Hamid and B. Kapralos, "A Review of Spatial Sound for Virtual Environments and Games with Graphics Processing Units", The Open Virtual Reality Journal, Volume 1, pp. 8-17, 2009.

[4] H. Yeh, R. Mehra, Z. Ren, L. Antani, D. Manocha and M. Lin, "Wave-ray coupling for interactive sound propagation in large complex scenes", ACM Transactions on Graphics (TOG) - Proceedings of ACM SIGGRAPH Asia 2013 TOG Homepage archive, Volume 32, pp. 165, Issue 6, 2013.

[5] C. Lauterbach, A. Chandak and D. Manocha, "Interactive sound rendering in complex and dynamic scenes using frustum tracing", IEEE Transactions on Visualization and Computer Graphics, Volume. 13, Issue 6, pp. 1672 1679, 2007.

[6] A. Sterling and M. C. Lin, "Interactive modal sound synthesis using generalized proportional damping”, I3D " 16 Proceedings of the 20th ACM SIGGRAPH Symposium on Interactive 3D Graphics and Games, pp. 7986, 2016.

[7] M. Labschütz, "Realistic real-time audio rendering in virtual environments", cg.tuwien.ac.at, 2011.

[8] M. Vorländer, "Auralization: Fundamentals of Acoustics", Modelling, Simulation, Algorithms, and Acoustic Virtual Reality, Springer-Verlag, Berlin, pp. 335, ISBN: 978-3-540-48829-3, 2008.

[9] T. Funkhouser, N. Tsingos, and J. M. Jot, "Survey of Methods for Modeling Sound Propagation in Interactive Virtual Environment Systems", Citeseer, 2003.

[10] C. Schissler, R. Mehra and D. Manocha, "High-order diffraction and diffuse reflections for interactive sound propagation in large environments", ACM Transactions on Graphics (TOG) - Proceedings of ACM SIGGRAPH, Volume 33, pp 12, Issue 4, 2014.

[11] R. Mehra and D. Manocha, "Wave-based sound propagation for VR applications", VR Workshop: Sonic Interaction in Virtual Environments (SIVE), pp 41-46, 2014.

[12] N.Atalla and R.J.Bernhard, "Review of numerical solutions for lowfrequency structural-acoustic problems", Applied Acoustics, Volume 43, Issue 3, pp 271-294, 1994.

[13] KJ Bathe, "Finite element procedures". Publisher: Prentice Hall, Pearson Education, Inc., First Edition, ISBN 978-0-9790049-5-7, 1996.

[14] L. Savioja, T. Rinne and T. Takala, "Simulation of room acoustics with a 3D finite difference mesh", Proceedings of Intl. Computer Music Conf. (ICMC94), pp. 463-466, 1994.

[15] N. Tsingos, C. Dachsbacher, S.Lefebvre and M. Dellepiane, "Instant sound scattering", EGSR'07 Proceedings of the 18th Eurographics conference on Rendering Techniques, pp. 111-120, 2007. 
[16] D. Quinn, "A finite element method for computing sound propagation in ducts containing flow", 5th Aeroacoustics Conference, https://doi. org/10.2514/6.1979-661, 1979.

[17] L. L. Thompson, "A review of finite-element methods for time-harmonic acoustics", The Journal of the Acoustical Society of America, Volume. 119, pp 1315-1330, Issue 3, 2006.

[18] Y.T. Chou and S.Y. Hsia, "Numerical Analysis of Indoor Sound Quality Evaluation Using Finite Element Method", Mathematical Problems in Engineering, Volume 2013, pp. 8, 2013.

[19] R. Mehra, N. Raghuvanshi, L. Antani, A. Chandak, S. Curtis and D. Manocha, "Wave-based sound propagation in large open scenes using an equivalent source formulation", ACM Transactions on Graphics (TOG), Volume 32, pp 13, Issue 2, 2013.

[20] L. Savioja, "Modeling techniques for virtual acoustics", Ph.D. dissertation, Helsinki University of Technology, Telecommunications Software and Multimedia Laboratory, Helsinki, Finland, 1999.

[21] R. Mehra, R, L. Antani, S. Kim and D. Manocha, "Source and Listener Directivity for Interactive Wave-Based Sound Propagation", IEEE Transactions on Visualization and Computer Graphics, Volume 20, Issue 4, pp. $495-503,2014$.

[22] L.H. Chen and D.G. Schweikert, "Sound Radiation from an Arbitrary Body", The Journal of the Acoustical Society of America, Volume 35, pp 1626-1632, Issue 10, 1963

[23] K. A. Cunefare and G. Koopmann, "A boundary element method for acoustic radiation valid for all wavenumbers", The Journal of the Acoustical Society of America, Volume 85, pp 39, Issue 1, 1989.

[24] J. Zhenlin M. Qiang and Z. Zhihua, "Application of the Boundary Element Method to Predicting Acoustic Performance of Expansion Chamber Mufflers With Mean Flow", Journal of Sound and Vibration, Volume 173, Issue 1, pp 57-71, 1994.

[25] B. F. G. Katz, "Boundary element method calculation of individual headrelated transfer function. I. Rigid model calculation", The Journal of the Acoustical Society of America, Volume 110, pp 2440-2448, Issue 5, 2001.

[26] W. Haijun, L. Yijun and J. Weikang, "A fast multipole boundary element method for 3D multi-domain acoustic scattering problems based on the Burton-Miller formulation", Engineering Analysis with Boundary Elements, Volume 36, pp 779-788, Issue 5, 2012.

[27] R. Blumricha and D. Heimann, "A linearized Eulerian sound propagation model for studies of complex meteorological effects", The Journal of the Acoustical Society of America, Volume 112, pp 446, Issue 2, 2002.

[28] E.M. Salomons, R. Blumrich and D. Heimann, "Eulerian time-domain model for sound propagation over a finite-impedance ground surface. Comparison with frequency-domain models", Acta Acustica united with Acustica, Volume 88, pp 483-492, 2002.

[29] K. Heutschi, M. Horvath and J. Hofmann, "Simulation of Ground Impedance in Finite Difference Time Domain Calculations of Outdoor Sound Propagation", Acta Acustica united with Acustica, Volume 91, pp 35-40(6), 2005.

[30] V.E. Ostashev, D.K. Wilson, L. Liu, D.F. Aldridge, N.P. Symons and D. Marlin, "Equations for finite-difference, time-domain simulation of sound propagation in moving inhomogeneous media and numerical implementation", The Journal of the Acoustical Society of America, Volume 117, pp 503-517, Issue 2, 2005.

[31] S. Van Duyne and J. O. Smith. "The 2-d digital waveguide mesh", Applications of Signal Processing to Audio and Acoustics, pp 177-180 (1993).

[32] L. Savioja, T. Lokki and V. Välimäki, "The interpolated 3-D digital waveguide mesh method for room acoustic simulation and auralization", Conference: Joint Baltic-Nordic Acoustical Meeting, Volume 2, pp 48-52, 2002.

[33] D. T. Murphy, "The digital waveguide mesh: Applications in acoustic modeling and current research directions", The Journal of the Acoustical Society of America, Volume 116, pp 2562-2562 ,Issue 4, 2004.

[34] D. Manocha and M.C. Lin, "Interactive sound rendering", ComputerAided Design and Computer Graphics, 2009.

[35] C. Schissler and D. Manocha, "Interactive sound propagation and rendering for large multi-source scenes", ACM Transactions on Graphics (TOG), Volume 36, pp 4, Issue 1, 2017.

[36] N. Raghuvanshi, J. Snyder, R. Mehra, M. Lin and N. Govindaraju, "Precomputed wave simulation for real-time sound propagation of dynamic sources in complex scenes", ACM Transactions on Graphics, Volume 29, Issue 4, 2010.

[37] T. Lokki, L. Savioja, R. Vaananen, J. Huopaniemi and T. Takala, "Creating interactive virtual auditory environments", IEEE Computer Graphics and Applications, Volume. 22, pp 49 - 57, Issue 4, 2002.

[38] T. Lokk and L. Savioja, "State-of-the-art in auralization of concet hall models - What is still missing?", Baltic-Nordic Acoustics Meeting, 2008.

[39] E.; Tavazzi and F. Avanzini, "Distance rendering and perception of nearby virtual sound sources with a near-field filter model", Applied Acoustics, Volume 115, pp 61-73, 2017.

[40] C. Lauterbach, A. Chandak and D. Manocha, "Interactive sound rendering in complex and dynamic scenes using frustum tracing", IEEE Transactions on Visualization and Computer Graphics, Volume 13, pp 1672-1679, Issue 6, 2007.

[41] L. Savioja, "Real-time 3D finite-difference time-domain simulation of low-and mid-frequency room acoustics", Proc. of the 13th Int. Conference on Digital Audio Effects (DAFx-10), Graz, Austria, 2010.

[42] N. Raghuvanshi and J. Snyder, "Parametric wave field coding for precomputed sound propagation", ACM Transactions on Graphics (TOG) - Proceedings of ACM SIGGRAPH, Volume 33, Issue 4, pp 11, 2014.

[43] T. Funkhouser, I. Carlbom, G. Elko, G. Pingali, M. Sondhi and J.West, "A beam tracing approach to acoustic modeling for interactive virtual environments", SIGGRAPH '98 Proceedings of the 25th annual conference on Computer graphics and interactive techniques, pp 21-32, 1998.

[44] L. Savioja and U.P. Svensson, "Overview of geometrical room acoustic modeling techniques", The Journal of the Acoustical Society of America, Volume 138, pp 708-730, Issue 2, 2015.

[45] S. Lauri, H. Jyri, L. Tapio and V. Ritta, "Creating interactive virtual acoustic environments", JAES, Volume 47, Issue 9, pp 675-705, 1999.

[46] B.M. Gibbs and D.K. Jones, "A Simple Image Method for Calculating the Distribution of Sound Pressure Levels within an Enclosure", Acta Acustica united with Acustica, Volume 26, pp 24-32, 1972.

[47] F. Santon, "Numerical prediction of echograms and of the intelligibility of speech in rooms", The Journal of the Acoustical Society of America, Volume 59, pp 1399-1405, Issue 6, 1976.

[48] J. B. Allen and D. A. Berkley, "Image method for efficiently simulating small-room acoustics", Acoustical Society of America, Volume 65, pp 943-950, Issue 4, 1979.

[49] J. Borish, "Extension of the Image Model to Arbitrary Polyhedra", The Journal of the Acoustical Society of America, Volume 75, pp 1827-1836, 1984.

[50] H. Lee and B-H. Lee, "An efficient algorithm for the image model technique", Applied Acoustics, Volume 24, pp 87-115, Issue 2, 1988.

[51] M. Vorländer, "Simulation of the transient and steady state sound propagation in rooms using a new combined ray tracing/image source algorithm", The Journal of the Acoustical Society of America, Volume 86, pp 172-178, 1989.

[52] R. Heinz, "Binaural room simulation based on an image source model with addition of statistical methods to include the diffuse sound scattering of walls and to predict the reverberant tail", Applied Acoustics, Volume 38, Issues 2-4, pp 145-159, 1993.

[53] E.A. Lehmann and A.M. Johansson, "Prediction of energy decay in room impulse responses simulated with an image-source model", The Journal of the Acoustical Society of America, Volume 124, pp 269-277, Issue 1, 2008.

[54] S. G. McGovern, "Fast image method for impulse response calculations of box-shaped rooms", Applied Acoustics, Volume. 70, Issue 1, pp 182-189, 2009.

[55] O. André, C. Guilherme, D. Paulo, M. D. Thomas, V. José, M. Catarina and S. Jorge, "Real-time dynamic image-source implementation for auralization", Proceedings of the 16th International Conference on Digital Audio Effects. 16th International Conference on Digital Audio Effects, pp 368-372, 2013.

[56] P. Charalampous and D. Michael, "Tree Traversal Algorithms for Real Time Sound Propagation Calculation", 55th International Conference: Spatial Audio, pp 4-1, 2014.

[57] P. Svensson, "The Early History of Ray Tracing in Room Acoustics", Modern Acoustics and Signal Processing, chapter 2, Part of the series Modern Acoustics and Signal Processing, pp 15-31, 2015.

[58] A. Krokstad, S. Strom and S. Sørsdal, "Calculating the acoustical room 
response by the use of a ray tracing technique", Journal of Sound and Vibration, Volume 8, pp 118-125, Issue 1, 1968.

[59] A. Kulowski, "Error investigation for the ray tracing technique", Applied Acoustics, Volume 15, pp 263-274, Issue 4, 1982.

[60] A. Kulowski, "Algorithmic representation of the ray tracing technique", Applied Acoustics, Volume 18, pp 449-469, Issue 6, 1985.

[61] H. Lehnert, "Systematic errors of the ray-tracing algorithm", Applied Acoustics, Volume 38, pp 207-221, Issues 2-4, 1993.

[62] D.V. Maercke and J. Martin, "The prediction of echograms and impulse responses within the Epidaure software", Applied Acoustics, Volume 38, pp 93-114, Issues 2-4, 1993.

[63] K.M. Li, S. Taherzadeh and K. Attenborough, “An improved ray-tracing algorithm for predicting sound propagation outdoors", The Journal of the Acoustical Society of America, Volume 104, pp 2077-2083, Issue 4, 1998.

[64] W. Mueller and F. Ullmann, "A scalable system for 3D audio ray tracing", Multimedia Computing and Systems - Italy, IEEE, 1999.

[65] A. Alpkocak and K. Sis, "Computing Impulse Response of Room Acoustics Using the Ray-Tracing Method in Time Domain", Archives of Acoustics, Volume 35, pp 505-519, Issue 4, 2010.

[66] M. Dreher, G. Dutilleux and F. Junker, "Optimized 3D ray tracing algorithm for environmental acoustic studies", Proceedings of the Acoustics 2012 Nantes Conference, 2012.

[67] M. Okada, T. Onoye and W. Kobayashi, "A Ray Tracing Simulation of Sound Diffraction Based on the Analytic Secondary Source Model", IEEE Transactions on Audio, Speech, and Language Processing, Volume 20, pp $2448-2460$, Issue 9, 2012.

[68] M. Taylor, A. Chandak, Q. Mo, C. Lauterbach, C. Schissler and D. Manocha, "Guided Multiview Ray Tracing for Fast Auralization", IEEE Transactions on Visualization and Computer Graphics, Volume 18, pp 1797 - 1810, Issue 11, 2012.

[69] Q. Mo, H. Yeh and D. Manocha, "Tracing Analytic Ray Curves for Light and Sound Propagation in Non-linear Media", IEEE Transactions on Visualization and Computer Graphics, Volume 22, pp 2493 - 2506, Issue 11, 2016.

[70] Q. Mo, H. Yeh, M. Lin and D. Manocha, "Analytic ray curve tracing for outdoor sound propagation”, Applied Acoustics, Volume 104, pp 142-151, 2016.

[71] C. Schissler and D. Manocha, "Adaptive impulse response modeling for interactive sound propagation", Proceedings of the 20th ACM SIGGRAPH Symposium on Interactive 3D Graphics and Games, pp 71-78, 2016.

[72] P. Charalampous and D. Michael, "Sound propagation in 3D spaces using computer graphics techniques", Virtual Systems \& Multimedia, 2015.

[73] T. Lewers, "A combined beam tracing and radiant exchange computer model of room acoustics", Applied Acoustics, Volume 38, pp 161-178, Issues 2-4, 1993.

[74] T. Funkhouser, P. Min and I. Carlbom, "Real-time acoustic modeling for distributed virtual environments", Proceedings of the 26th annual conference on Computer graphics and interactive techniques, pp 365-374, 1999.

[75] T. Funkhouser, I. Carlbom, G. Elko, G. Pingali, M. Sondhi and J. West, "Interactive Acoustic Modeling of Complex Environments", The Journal of the Acoustical Society of America, Volume 105, pp 1357-1358, Issue $2,1999$.

[76] N. Tsingos, T. Funkhouser, A. Ngan and I. Carlbom, "Modeling acoustics in virtual environments using the uniform theory of diffraction", Proceedings of the 28th annual conference on Computer graphics and interactive techniques, pp 545-552, 2001.

[77] T. Funkhouser, N. Tsingos, I. Carlbom, G. Elko, M. Sondhi, J. E West, G. Pingali, P. Min and A. Ngan, "A beam tracing method for interactive architectural acoustics", The Journal of the Acoustical Society of America, Volume 115, pp 739-756, Issue 2, 2003.

[78] R. Ajaj, L. Savioja and C. Jacquemin, "Software platform for real-time room acoustic visualization", Proceedings of the 2008 ACM symposium on Virtual reality software and technology, pp 247-248, 2008.

[79] S. Laine, S. Siltanen, T. Lokki and L. Savioja, "Accelerated beam tracing algorithm", Applied Acoustics, Volume 70, Issue 1, pp 172-181, 2009.

[80] F. Antonacci, M. Foco, A. Sarti and S. Tubaro, "Fast Tracing of Acoustic Beams and Paths Through Visibility Lookup", IEEE Transactions on Audio, Speech, and Language Processing, Volume 16, Issue 4, pp 812 $824,2008$.
[81] F. Antonacci, A. Sarti and S. Tubaro, "Two-Dimensional Beam Tracing from Visibility Diagrams for Real-Time Acoustic Rendering", Proceeded of the 7th Int. Conference on Digital Audio Effects, 2010.

[82] T. Funkhouser, J.-M. Jot and N. Tsingos, "Sounds Good to Me!" Computational Sound for Graphics, Virtual Reality, and Interactive Systems, SIGGRAPH 2002 Course Notes, 2002.

[83] P. Svensson and U.R. Kristiansen, "Computational Modelling and Simulation of Acoustic Spaces", 22nd International Conference: Virtual, Synthetic, and Entertainment Audio, 2002.

[84] A. Rajkumar, B. F. Naylor, F. Feisullin and L. Rogers, "Predicting RF coverage in large environments using ray-beam tracing and partitioning tree represented geometry", Wireless Networks, Volume 2, pp 143-154, Issue 2, 1996.

[85] E. Deines, M. Bertram, J. Mohring, J. Jegorovs, F. Michel, H. Hagen and G.M. Nielson, "Comparative Visualization for Wave-based and Geometric Acoustics", IEEE Transactions on Visualization and Computer Graphics, Volume. 12, pp 1173 - 1180, Issue 5, 2006.

[86] Z. Yamauti, "The light flux distribution of a system of interreflecting surfaces", J. Opt. Soc. Am., Volume 13, pp 561-571, Issue 5, 1926.

[87] E.M. Nosal, "Room sound field prediction by acoustical radiosity master thesis", 2002.

[88] J. Shi, A. Zhang, J. Encarnacao and M. Göbel, "A modified radiosity algorithm for integrated visual and auditory rendering", Computers \& Graphics, Volume 17, pp 633-642, Issue 6, 1993.

[89] N. Tsingos and J.D. Gascuel, "A general model for the simulation of room acoustics based on hierarchical radiosity", SIGGRAPH'97 technical sketch, 1997.

[90] N. Tsingos and J.D. Gascuel, "Acoustic simulation using hierarchical timevarying radiant exchanges", iMAGIS-GRAVIR/IMAG - Citeseer, 1997.

[91] E.M. Nosal and M. Hodgson, "Preliminary experimental validation of the radiosity technique for predicting room sound fields", Citeseer.

[92] E.M. Nosal, M. Hodgson and I. Ashdown, "Improved algorithms and methods for room sound-field prediction by acoustical radiosity in arbitrary polyhedral rooms", The Journal of the Acoustical Society of America, Volume 116, pp 970-980, Issue 2, 2004.

[93] J. Kang, Application of radiosity method in acoustic simulation, ICA, 2004.

[94] S. Siltanen, T. Lokki, S. Kiminki and L. Savioja, "The Room Acoustic Rendering Equation", The Journal of the Acoustical Society of America, Volume 122, pp 1624-1635, Issue 3, 2007.

[95] R.T. Muehleisen, "Six Surface Steady State Acoustic Radiosity", Architectural Engineering Conference (AEI), 2008.

[96] S.M. Pasareanu, "A numerical hybrid method for modeling outdoor sound propagation in complex urban environments", master thesis, URL: http:// hdl.handle.net/10919/47601, 2014.

[97] M. Sikora, "Algorithms for simulations of sound and ultrasound propagation in complex dispersive environments", Proceedings Elmar 2004. 46th International Symposium, 2004.

[98] E. Stavrakis, N. Tsingos and P. Calamia, "Topological Sound Propagation with Reverberation Graphs", Acta Acustica united with Acustica, Volume 94, pp 921-932, Number 6, 2008.

[99] M. Taylor, A. Chandak, L. Antani and D. Manocha, "RESound: Interactive Sound Rendering for Dynamic Virtual Environments", MM '09 Proceedings of the 17th ACM international conference on Multimedia, pp 271-280, 2009

[100] A. Pohl and U.M. Stephenson, "A Combination of the Sound Particle Simulation Method and the Radiosity Method", Building Acoustics, Volume 18, pp 97-122, Issue 1/2, 2011.

[101] S. Pelzer, B. Masiero and M. Vorländer, "3D Reproduction of Room Auralizations by Combining Intensity Panning", Crosstalk Cancellation and Ambisonics, Proc. of the EAA Joint Symposium on Auralization and Ambisonics, Berlin, pp 182-188, 2014.

[102] R. Mehra, A. Rungta, A. Golas, M. Lin and D. Manocha, "WAVE: Interactive Wave-based Sound Propagation for Virtual Environments", IEEE Transactions on Visualization and Computer Graphics, Volume 21, pp 434 - 442, Issue 4, 2015.

[103] P. Charalampous and D. Michael, "Improved hybrid algorithm for real time sound propagation using intelligent prioritization", Electrotechnical Conference (MELECON), 18th Mediterranean, 2016.

[104] I. Podkosova, M. Urbanek and H. Kaufmann, "A Hybrid Sound Model 
for 3D Audio Games with Real Walking", Proceedings of the 29th International Conference on Computer Animation and Social Agents, pp 189-192, 2016.

[105] A. Rungta, C. Schissler, R. Mehra, C. Malloy, M. Lin and D. Manocha, "SynCoPation: Interactive Synthesis-Coupled Sound Propagation", IEEE Transactions on Visualization and Computer Graphics, Volume 22, pp 1346 - 1355, Issue 4, 2016.

[106] B. Smus, "Web Audio API". O’Reilly Media, Inc, 2013.

[107] R. Spuy, "Sound with the Web Audio API. In Advanced Game Design with HTML5 and JavaScript". IEEE, 2015.

[108] B. Smus, "Retrieved from Developing Game Audio with the Web Audio API", Available online: http://www.html5rocks.com/en/tutorials/ webaudio/games/ (accessed May 2015).

[109] H. Choi and J. Berger, "WAAX: Web Audio API eXtension”, International Conference on New Interfaces for Musical Expression, KAIST, Daejeon, Korea, 2013

[110] C. Pike, P. Taylour and F. Melchior, "Delivering Object-Based 3D Audio Using The Web Audio API And The Audio Definition Model", WAC - 1st Web Audio Conference, 2015.

[111] H. Rawlinson, N. Segal and J. Fiala, "Meyda: an audio feature extraction library for the Web Audio API", WAC - 1st Web Audio Conference, 2015.

[112] J. Kleimola and O. Larkin, "Web Audio Modules", 12th Sound and Music Computing Conference, Maynooth, Ireland, 2015.

[113] C. Pendharkar, P. Bäck and L. Wyse, "Adventures in scheduling, buffers and parameters: Porting a dynamic audio engine to Web Audio", WAC 1st Web Audio Conference, 2015.

[114] N. Schnell, V. Saiz, K. Barkati and S. Goldszmidt, "Of Time Engines and Masters An API for Scheduling and Synchronizing the Generation and Playback of Event Sequences and Media Streams for the Web Audio API", WAC - 1st Web Audio Conference, 2015.

[115] A. Mahadevan, J. Freeman and B. Magerko, "EarSketch: Teaching computational music remixing in an online Web Audio based learning environment", WAC - 1st Web Audio Conference, 2015.

[116] A. Stamoulias, E. Lakka and A.G. Malamos, “"Wrapping X3DOM around Web Audio API", International Journal of Interactive Multimedia and Artificial Intelligence, Volume 3, pp 36-46, Number 5, 2015.

[117] M. Cohen, J. Villegas and W. Barfield, "Special issue on spatial sound in virtual, augmented, and mixed reality environments", Virtual Reality, Volume 19, pp 147-148, Issue 3, 2015.

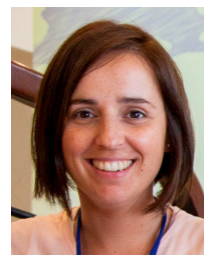

\section{E. Lakka}

Eftychia Lakka was born in 1982. She received her BSc degree in Computer Science from the University of Ioannina, Greece (2006) and her M.Sc. in Synthesis of Images and Graphic Designs from the University of Limoges, France (2011). Since October 2016, she has started the MPhil/PhD in School of Computing and Mathematics within the Faculty of Computing, Engineering and Science in the University of South Wales, UK. She has been as Research Associate at the Advanced Knowledge, Image \& Information Systems Laboratory, dept. of Informatics, T.E.I. of Athens (2010-2014) and at the Multimedia Content Laboratory, dept. of Informatics Engineering, T.E.I. of Crete (2014- 2015). Since April 2016, she has joined Information Technologies Institute of Centre for Research and Technology as research assistant. Her research interests include 3D computer games, 3D sound propagation, graphics and multimedia web applications.

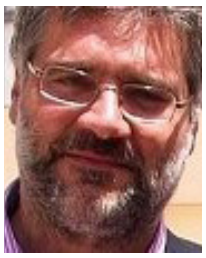

\section{A. G. Malamos}

Athanasios G. Malamos was born in 1969. He received his BSc degree in Physics from the University of Crete (1992) and his $\mathrm{PhD}$ from the Technical University of Crete in 2000. From 1997 to 2002 he was a Research Assistant and a researcher in the ICCS National Technical University of Athens. Since 2002 he is with the Technological Educational Institute of Crete at the Department of Informatics Engineering as an Assistant Professor (2002-2006) and as an Associate Professor (2006 until present). He is the coordinator of the Multimedia Content Lab, and has supervised many research projects granted with EU and National research funds. He has served as program committee member and reviewer for several international conferences and workshops. Dr. Malamos is a reviewer for IEEE, Springer as other international journals. He is member of the IEEE Computer Society, ACM SIGGRAPH and the WEB3D consortium. His research interests include multimedia semantics, AI and graphics.

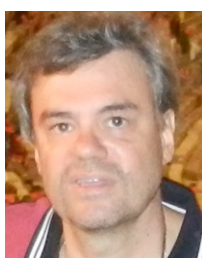

\section{K. G. Pavlakis}

Konstantinos G. Pavlakis got his B. Sc. in Physics, the M. Sc. on space physics and the $\mathrm{Ph}$. D. on radiation transfer and astrophysical Masers from the department of Physics, University of Crete. He is currently a lecturer in the field of general physics in the department of Mechanical Engineering of the Technological Education Institute of Crete. His scientific interests include modeling of radiation transfer in atmospheres, the study of climate change, and environmentalatmospheric physics. He has published 27 scientific papers in refereed journals and 11 papers in conference proceedings taking part in research projects as a researcher in the University of Leeds, the University of Crete, the Foundation of Research and Technology and the Technological Education Institute of Crete.

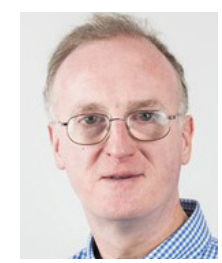

J. A. Ware

J. Andrew Ware is a Professor of Computing at the University of South Wales where he is engaged in both teaching and research. His main research area is Applied Artificial Intelligence, much of which is carried out in collaboration will business and industrial partners. Andrew has successfully supervised over $30 \mathrm{PhD}$ students. He is a Fellow of the British Computer Society and Senior Fellow of the Higher Education Academy. Andrew is also Regional Director of Technocamps, an innovative and progressive initiative aimed at increasing the number of young people studying Computer Science at school and university. 